\title{
Effects on inflammatory responses by the sphingoid base 4,8-sphingadienine
}

\author{
EVELIEN ROZEMA ${ }^{1}$, MARKUS BINDER $^{2}$, MURTY BULUSU $^{2}$, \\ VALERY BOCHKOV ${ }^{2}$, GEORG KRUPITZA ${ }^{3}$ and BRIGITTE KOPP ${ }^{1}$ \\ ${ }^{1}$ Department of Pharmacognosy, University of Vienna; ${ }^{2}$ Department of Vascular Biology and \\ Thrombosis Research, Center for Biomolecular Medicine and Pharmacology, and ${ }^{3}$ Institute \\ of Clinical Pathology, Medical University of Vienna, A-1090 Vienna, Austria
}

Received March 4, 2012; Accepted May 4, 2012

DOI: $10.3892 /$ ijmm.2012.1035

\begin{abstract}
Sphingolipids (SLs) are a class of lipids, which are structural cell components involved in the regulation of cellular processes such as cell proliferation, differentiation, apoptosis and inflammation. Dietary SLs are enzymatically hydrolyzed in the gut lumen into metabolites, namely ceramides and sphingoid bases. The sphingoid base 4,8 -sphingadienine $(4,8-$ $\mathrm{SD})$ is the metabolite of glucocerebrosides derived from plants that are part of the human diet. The present findings provide insight into the effects of 4,8-SD on inflammatory responses that may be of nutritional and therapeutic benefit. We demonstrated that 4,8-SD significantly inhibited tumor necrosis factor- $\alpha$ (TNF- $\alpha$ )- and lipopolysaccharide (LPS)-induced expression of IL-8 and E-selectin in human endothelial cells in a dose-dependent manner. The anti-inflammatory effect was observed at significantly lower concentrations of 4,8-SD compared those affecting cell viability as judged by the LDH and WST-1 assays.
\end{abstract}

\section{Introduction}

Sphingolipids (SLs) are a class of lipids, which are structural cell components involved in the regulation of cellular processes such as cell proliferation, differentiation, apoptosis and inflammation (1-5). The basic structure of SLs contains a sphingoid base that is $N$-acylated with a fatty acid and C-1 linked to a

Correspondence to: Dr Brigitte Kopp, Department of Pharmacognosy, University of Vienna, Althanstrasse 14, A-1090 Vienna, Austria

E-mail: brigitte.kopp@univie.ac.at

Abbreviations: CC, column chromatography; CD, circular dichromism; ELISA, enzyme-linked immunosorbent assay; FCS, fetal calf serum; GC-MS, gas chromatography mass spectrometry; GluCer, glucocerebrosides; IL-8, interleukin 8; LPS, lipopolysaccharide; NMR, nuclear magnetic resonance; 4,8-SD, 4,8-sphingadienine; SL, sphingolipid; TNF- $\alpha$, tumor necrosis factor- $\alpha$

Key words: 4,8-sphingadienine, sphingoid base, inflammation, cell viability polar head group. SLs are found in eukaryotes and prokaryotes and are well studied in mammals (6). The mammalian SL metabolites ceramide, sphingosine and sphingosine 1-phosphate have drawn attention as bioactive signaling molecules.

Inflammation is important in the healing processes of tissue injury or infections. Tumor necrosis factor- $\alpha$ (TNF- $\alpha$ ) and lipopolysaccharide (LPS) are essential pro-inflammatory stimuli involved in the pathogenesis of chronic inflammatory diseases. Chronic inflammation, however, is harmful to tissue and is considered to underlie the onset and development of a wide variety of pathological responses causing tissue damage. Diseases such as cancer, atherosclerosis, insulin resistance and diabetes are associated with chronic inflammation (7). Therefore the timely shut down of inflammatory processes is of utmost importance to maintain individual health.

Dietary SLs, such as glucocerebrosides (GluCer) derived from plants, are enzymatically hydrolyzed by brush border enzymes in the gut lumen into metabolites namely ceramides and sphingoid bases. This results in the uptake of the derived ceramides and sphingoid bases by intestinal epithelial cells (8-10). Sphingoid bases or 'sphingosines' occur in a great variety in type and chain length, such as sphingosine (d18:1) in mammals, and sphingomyelin $(\mathrm{d} 16: 1)$ in milk $(11,12)$. Several studies have reported on SLs regulating inflammatory responses at multiple levels $(4,13)$.

Research of anti-inflammatory effects of plant GluCer and sphingoid bases is very limited. 4,8-Sphingadienine (4,8-SD) (d18:2) is the sphingoid backbone of the characteristic GluCer present in the Araceae species Arisaema amurense Maxim. (14) and Pinellia ternata (Thunb.) Breit., as well as in plants in human diet such as spinach, soybean and eggplant $(11,15)$.

To the best of our knowledge, this is the first time the effect of the plant-derived sphingoid base on inflammatory responses was tested by investigation of the regulation of TNF- $\alpha$ - and LPS-induced expression of IL-8 and E-selectin.

\section{Materials and methods}

Materials. Fetal calf serum (FCS) was purchased from HyClone (Logan, UT, USA) and TNF- $\alpha$ was from Genzyme (Cambridge, MA, USA). BAY 11-7085 (BAY) (purity $\geq 98 \%$ ), medium 199, o-phenylenediamine and LPS from $E$. coli serotype 055:B5 


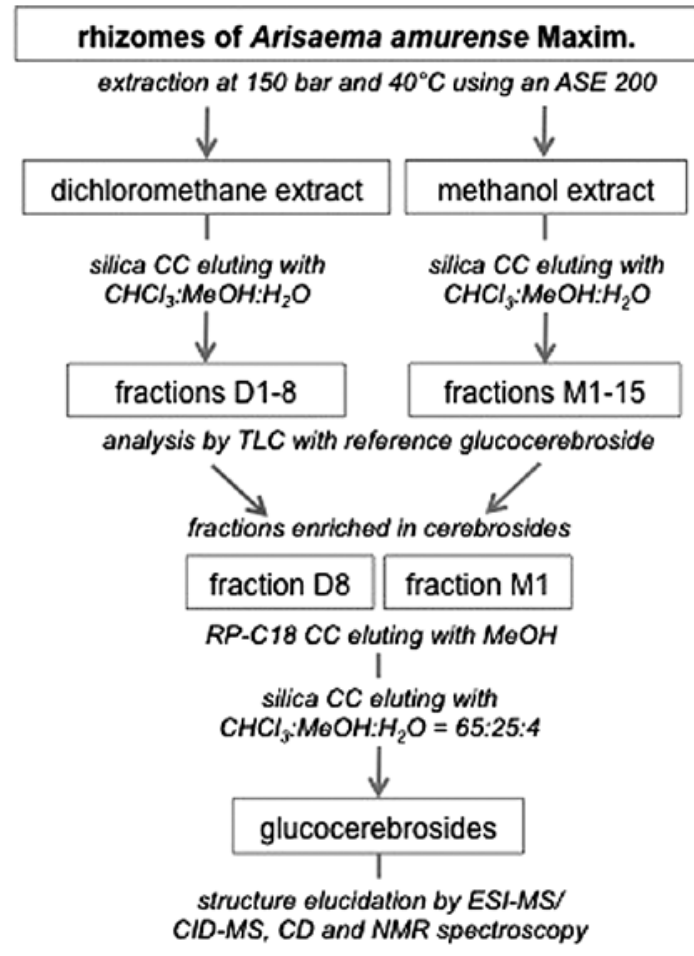

Figure 1. Isolation of cerebrosides from rhizomes of Arisaema amurense Maxim. (Araceae). CC, column chromatography; CD, circular dichromism; ESI-MS/CID-MS, electrospray ionization collision induced dissociation tandem mass spectrometry; NMR, nuclear magnetic resonance.

were purchased from Sigma-Aldrich (Vienna, Austria). The 4,8-SD obtained after hydrolysis of GluCer was isolated from Arisaema amurense Maxim. (Araceae) and purified by silica column chromatography (CC). Polyclonal antibodies were purchased from R\&D Systems (Minneapolis, MN, USA) and peroxidase-conjugated secondary antibodies from Amersham Life Science (Amersham, UK). For extraction, fractionation and isolation by $\mathrm{CC}$ solvents of highest available purity were used (VWR, Vienna, Austria). All other chemicals were obtained from Sigma-Aldrich.

Plant material. Dried, processed rhizomes of Arisaema amurense Maxim. were purchased from Plantasia (Oberndorf, Austria). A voucher specimen, encoded ER-I, was deposited at the Department of Pharmacognosy, University of Vienna.

Isolation of GluCer and 4,8-SD. The dried, processed rhizomes of $A$. amurense $(1.9 \mathrm{~kg})$ were pulverized and extracted by dichloromethane and subsequently $1.4 \mathrm{~kg}$ of this material was extracted with methanol. The dried extracts were chromatographed over a silica column eluted by $\mathrm{CHCl}_{3}: \mathrm{MeOH}: \mathrm{H}_{2} \mathrm{O}$ 98:2:1 to $60: 38: 8.5$ to obtain fractions D1-8 for the dichloromethane extract $(9.8 \mathrm{~g})$ and eluted by $\mathrm{CHCl}_{3}: \mathrm{MeOH}: \mathrm{H}_{2} \mathrm{O}$ 70:22:3.5 to 60:40:10 for the methanol extract $(10.7 \mathrm{~g})$ to obtain fractions M1-15. GluCer were isolated from fractions D8 (3.6 g) and M1 (2.5 g) by RP C-18 $\mathrm{CC}$ with methanol as mobile phase and subsequent silica $\mathrm{CC}$ with $\mathrm{CHCl}_{3}: \mathrm{MeOH}: \mathrm{H}_{2} \mathrm{O}(65: 25: 4)$ as mobile phase (Fig. 1). A mixture of GluCer $(40 \mathrm{mg})$ was hydrolyzed in methanolic $1 \mathrm{M}$ $\mathrm{HCl}$ for $7 \mathrm{~h}$ at $70^{\circ} \mathrm{C}$ under reflux. The hydrolyzed sample was neutralized and evaporated to dryness. The liberated sphin-

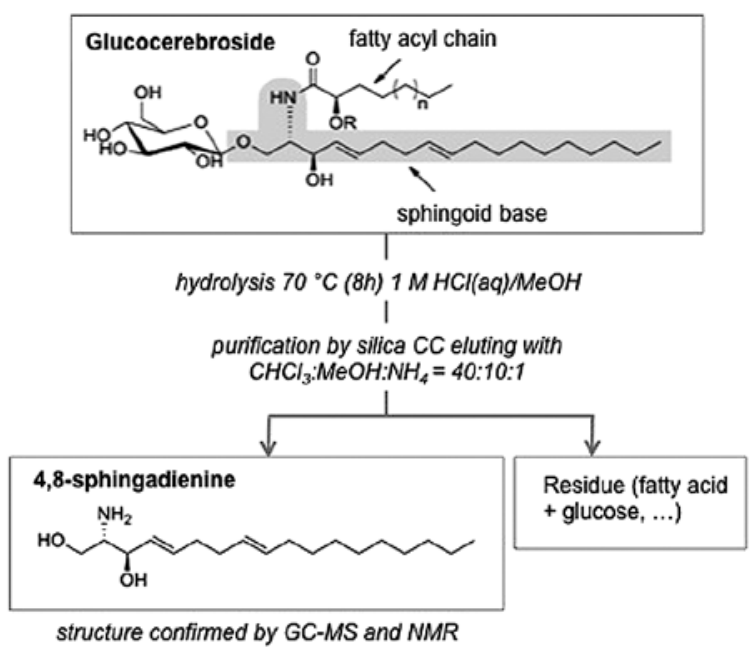

Figure 2. Hydrolysis of cerebrosides and isolation of the sphingoid base 4,8 -sphingadienine (4,8-E,E-SD only depicted of the isomeric mixture).

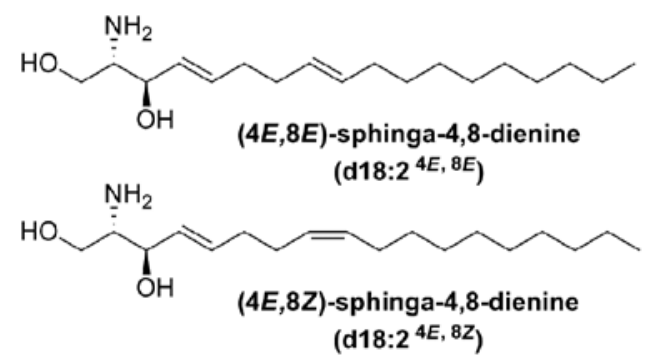

Figure 3. Structures of the isomers of $4 E, 8 E$-sphingadienine and $4 E, 8 Z$ sphingadienine.

goid base was purified by silica CC with $\mathrm{CHCl}_{3}: \mathrm{MeOH}: \mathrm{NH}_{4}$ (40:10:1) as mobile phase (Fig. 2), with a yield of $5.5 \mathrm{mg}$.

$G C-M S$ and NMR analysis. The pure compound was further converted to the $N$-acetyl-di- $O$-trimethylsilyl derivative and analyzed by GC-MS and NMR and identified as 4,8-sphingadienine (4,8-SD) (Fig. 3). The amide group was $\mathrm{N}$-acetylated with acetic anhydride: $\mathrm{MeOH}(\mathrm{v} / \mathrm{v} 1: 4)$ overnight at $25^{\circ} \mathrm{C}$. The sample was brought to dryness and further derivatized by BSTFA and TMCS (v/v 99:1) and incubated at $50^{\circ} \mathrm{C}$ for $30 \mathrm{~min}$. The analyses were performed on an Agilent Technologies 6890N Network GC equipped with an Agilent Technologies 5973 inert Mass selective Detector and a CombiPAL autosampler (CTC Analytics). The column used was a DB-5 with dimensions of $30 \mathrm{~m} \times 0.25 \mathrm{~mm}$ (inner diameter), $0.23 \mu \mathrm{m}$ (film thickness) (Agilent Technologies). The software used was MSD Chemstation 2004.

NMR spectra were recorded on a Bruker Avance 500 NMR spectrometer. 4,8-SD was dissolved in $\mathrm{CDCl}_{3}(99.96$ atom $\%$ D). The ${ }^{1} \mathrm{H}$ and ${ }^{13} \mathrm{C}$ NMR spectra were operated at 500 and $125 \mathrm{MHz}$, respectively. The compound has a purity of at least $95 \%$ judging from the NMR-spectra.

4,8-SD was a brownish, yellow, amorphous solid. Electron impact mass spectrometry (EIMS) of the $\mathrm{N}$-acetyl-di- $O$ trimethylsilyl derivative showed ions at $\mathrm{m} / \mathrm{z} 73,174,309,334$, 378,468 (16). For biological testing 4,8-SD was dissolved in DMSO 

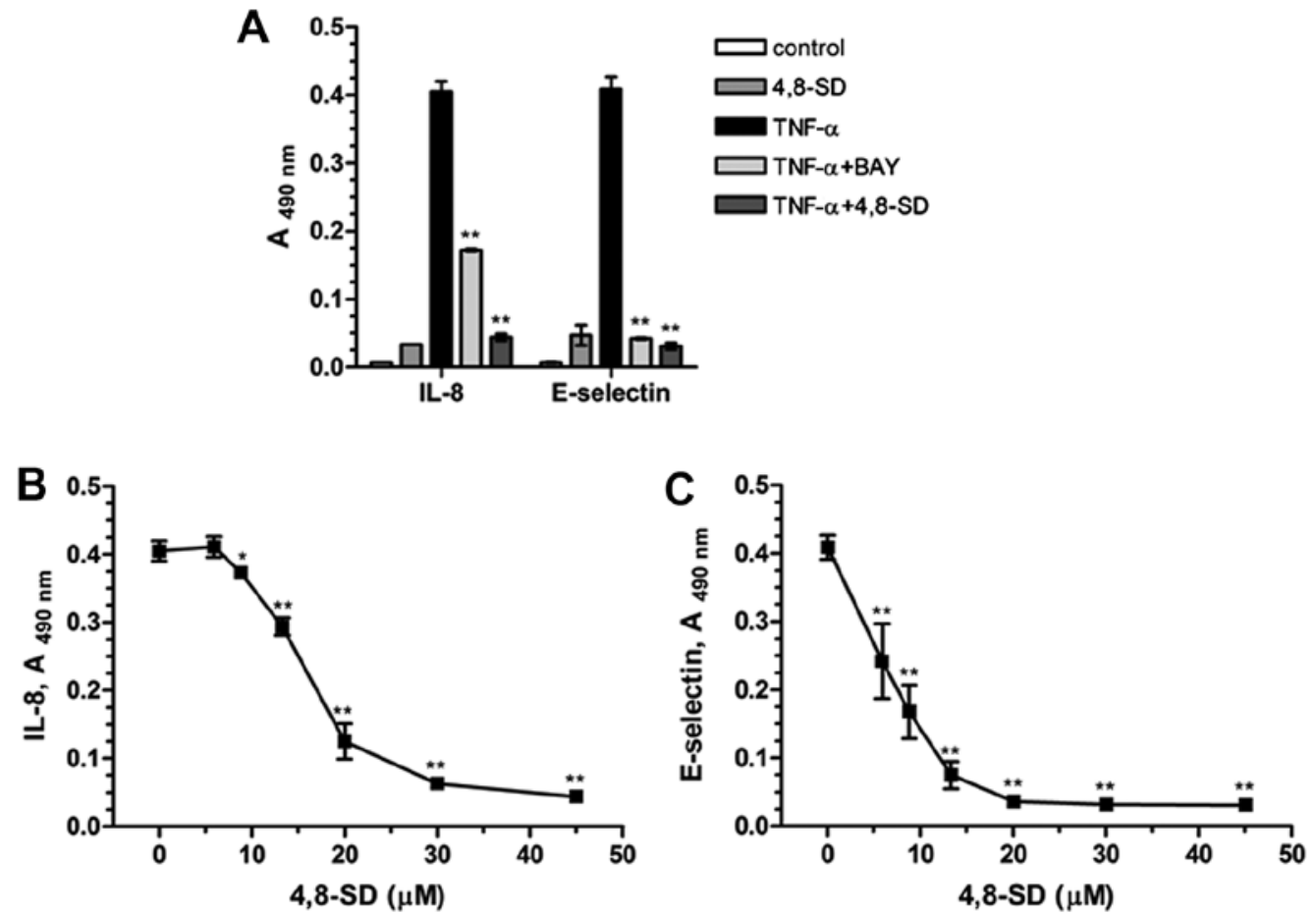

Figure 4. The sphingoid base 4,8-SD inhibits TNF- $\alpha$-induced expression of inflammatory adhesion molecules in HUVECtert cells. (A) HUVECtert cells were stimulated with TNF- $\alpha(100 \mathrm{ng} / \mathrm{ml})$ in the presence or absence of $4,8-\mathrm{SD}(30 \mu \mathrm{M})(6 \mathrm{~h}$ treatment). For comparison BAY $(5 \mu \mathrm{M})$ was used as a known antagonist of inflammation and cells incubated with $0.2 \%$ DMSO served as control in (A). TNF-induced cells incubated with $0.2 \%$ DMSO served as negative control in (B and C) and were set as zero. Dose-response curves of 4,8-SD inhibiting TNF- $\alpha$-induced upregulation of both (B) IL-8 and (C) E-selectin. Results are presented as mean values out of at least three experiments \pm SEM. Significantly different from the negative control (ANOVA): ${ }^{*} \mathrm{P}<0.05,{ }^{* *} \mathrm{P}<0.01$ compared to cells treated only with TNF- $\alpha$.

Cells. The study was performed using immortalized human umbilical vein endothelial (HUVECtert) cells (17). In contrast to primary HUVECs, HUVECtert have the advantage of an indefinite cell division potential. However, the tert transfection alters the cells' gene expression profile (18) affective the response to the dual treatment. HUVECtert were cultured in M199 medium supplemented with endothelial cell growth supplement (Technoclone), heparin, penicillin/streptomycin and 20\% FCS. The cells were grown in a humidified atmosphere at $37^{\circ} \mathrm{C}$ and $5 \% \mathrm{CO}_{2}$ and passaged twice a week using trypsin-EDTA solution. Experiments were performed using cells up to passage 5 .

Cell ELISA. HUVECtert cells ( $1 \times 10^{5}$ cells/well) were seeded in a 96-well plate and grown for 24 h overnight in a cell culture incubator. Monolayers of the grown HUVECtert cells were then treated with 4,8-SD or GluCer and agonists in medium 199 containing $5 \%$ FCS. $4,8-\mathrm{SD}(0-45 \mu \mathrm{M})$ or BAY $11-7082$ $(5 \mu \mathrm{M})$, which served as positive control, were added to the cells $20 \mathrm{~min}$ before the addition of TNF (100 ng/ml) or LPS (100 ng/ml). BAY 11-7085 is an anti-inflammatory agent acting by inhibiting NF- $\kappa \mathrm{B}$ thus decreasing expression of inflammatory molecules including E-selectin and IL-8 (19). The solvent vehicle $(0.2 \%$ DMSO) served as the negative control. After $6 \mathrm{~h}$, the medium was collected for quantification of IL-8, and the cells were washed and fixed with $4 \%$ glutaraldehyde for determination of E-selectin. Cell surface-expressed E-selectin was detected using corresponding antibodies, secondary peroxidase-conjugated antibodies and $o$-phenylenediamine as substrate essentially as described before (20). Concentrations of IL-8 in cell culture medium were determined using human
CXCL8/IL-8 ELISA DuoSet ELISA Development kit (R\&D Systems) and the TMB 2-Component Microwell Peroxidase Substrate kit (VWR International, Radnor, PA, USA). The absorbance was measured at $490 \mathrm{~nm}$.

Cell proliferation/viability assays. The HUVECtert cells $\left(1 \times 10^{5}\right.$ cells/well) were seeded in 96-well plates. After $24 \mathrm{~h}$ test compounds $(4,8$-SD or GluCer) or vehicle (DMSO) were added. Cell viability and metabolic activity were assessed after $6 \mathrm{~h}$ either by the LDH (Sigma Aldrich, St. Louis, MO, USA) or WST-1 (Roche Applied Science, Mannheim, Germany) colorimetric assays. The absorbance was measured at $340 \mathrm{~nm}$ after $55 \mathrm{~min}$ for the LDH assay at $450 \mathrm{~nm}$ for the WST1-assay.

Data and statistical analysis. Statistical analysis and calculation of $\mathrm{IC}_{50}$ values were performed using the Prism Software (ver. 4.03; GraphPad Software Inc., San Diego, CA, USA). Data were normalized to the mean value from three experiments of DMSO treated control. The data shown represent the mean values out of at least three experiments \pm SEM. Statistical significance was determined by a one-way analysis of variance combined with a Dunnett's multiple comparison post test. Results with $\mathrm{P}<0.05$ were considered significant.

\section{Results}

In our studies 4,8-SD (Fig. 3) blocked TNF- $\alpha$ - (Fig. 4) and LPS-induced (Fig. 5) upregulation of the inflammatory adhesion molecule E-selectin and the cytokine IL- 8 in HUVECtert cells. 4,8-SD showed this inhibitory effect in a 

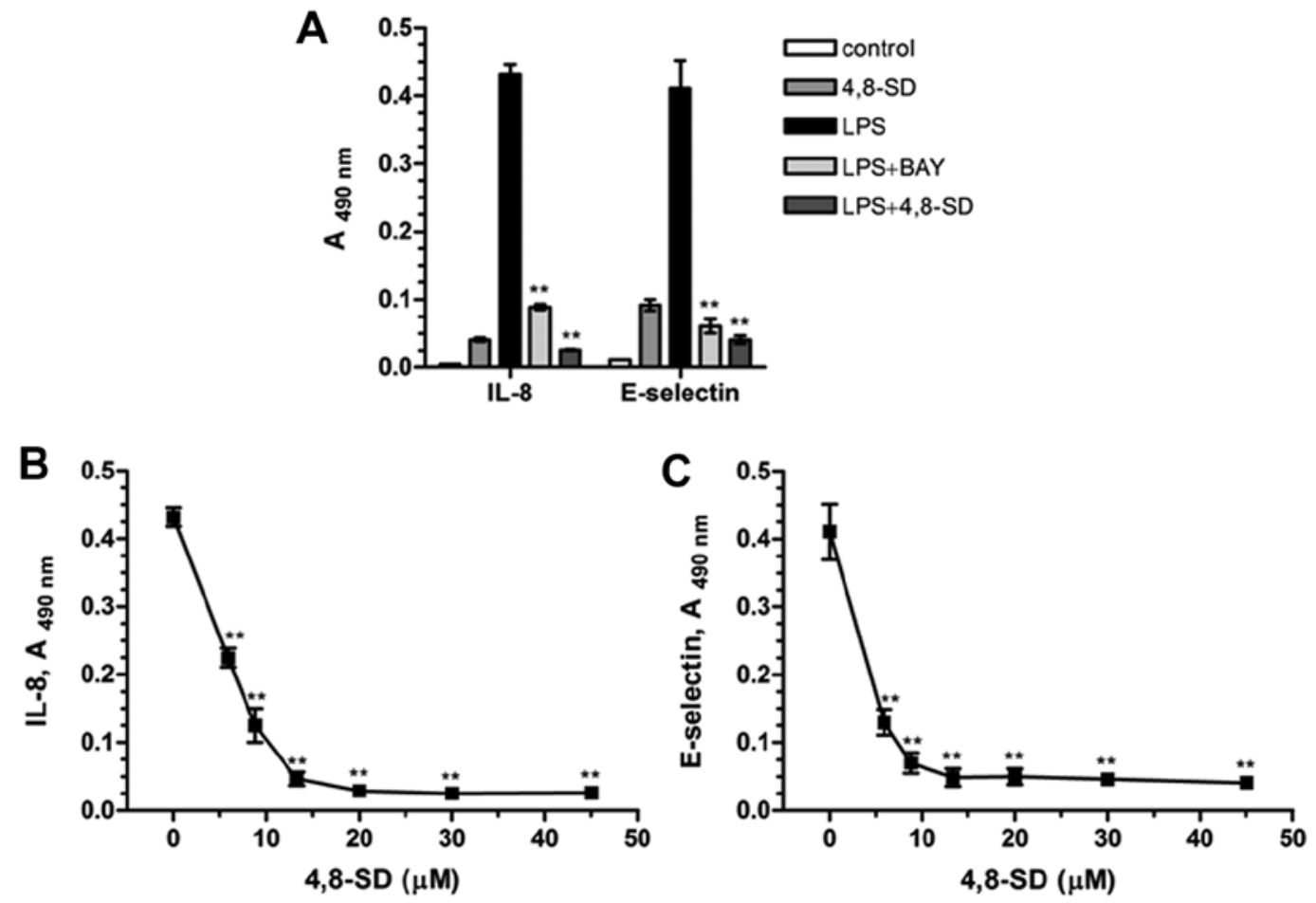

Figure 5. The sphingoid base 4,8-SD inhibits LPS-induced expression of inflammatory adhesion molecules in HUVECtert cells. (A) HUVECtert cells were stimulated with LPS $(100 \mathrm{ng} / \mathrm{ml})$ in the presence or absence of 4,8-SD $(30 \mu \mathrm{M})(6 \mathrm{~h}$ treatment). For comparison BAY $(5 \mu \mathrm{M})$ was used as a known antagonist of inflammation and cells incubated with 0.2\% DMSO served as control in (A). LPS-induced cells incubated with $0.2 \%$ DMSO served as negative control in (B and C) and were set to zero. (B and C) Dose-response curves of 4,8-SD (0-45 $\mu \mathrm{M})$ inhibits LPS-induced upregulation of both (B) IL-8 and (C) E-selectin. Significantly different from the negative control (ANOVA): ${ }^{* *} \mathrm{P}<0.05,{ }^{* *} \mathrm{P}<0.01$ compared to cells treated with LPS alone.
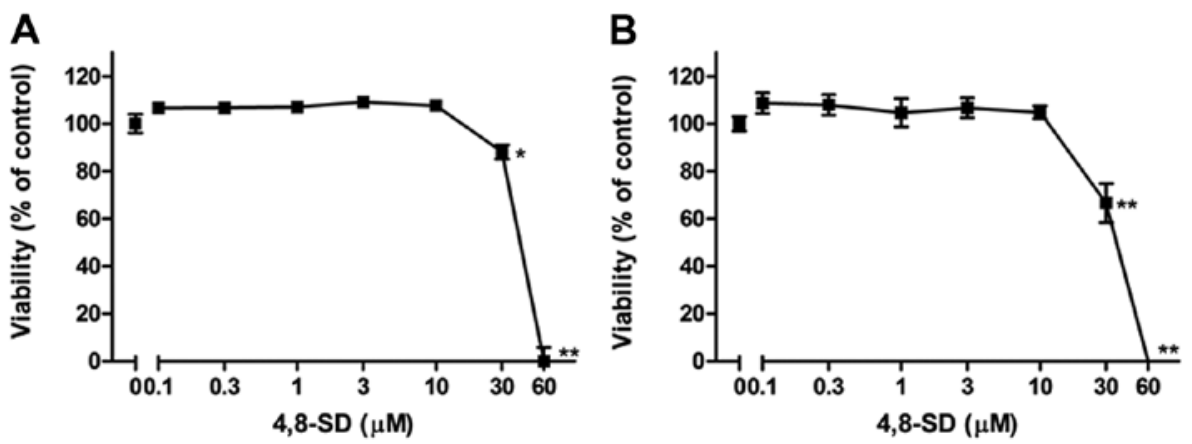

Figure 6. Cell viability of HUVECtert cells treated with 4,8-SD. Cell viability in HUVECtert cells after $6 \mathrm{~h}$ of treatment with 4,8-SD determined in the (A) LDH and (B) WST-1 assay. Data of cells treated with DMSO were set as $100 \%$. ${ }^{*} \mathrm{P}<0.05,{ }^{* *} \mathrm{P}<0.01$ compared to cells treated with DMSO (0).

concentration-dependent manner. Half-maximal inhibition of TNF- $\alpha$-induced upregulation of IL-8 (Fig. 4B) and E-selectin (Fig. 4C) was observed at 15.4 and $6.8 \mu \mathrm{M} 4,8$-SD, respectively. Half-maximal inhibition of LPS-induced upregulation of IL-8 (Fig. 5B) and E-selectin (Fig. 5C) was observed at 6.0 and $4.2 \mu \mathrm{M} 4,8$-SD, respectively. 4,8-SD exerted an inflammatory response by the unstimulated HUVECtert cells at the cytotoxic concentration of $30 \mu \mathrm{M}$, which exceeds the control (Figs. 4A and 5A).

In addition, it was investigated whether the treatment of the cells with 4,8-SD induced cell death that could influence TNF- $\alpha$ - and LPS-induced IL- 8 and E-selectin expression. To this end, cytotoxic effects of 4,8-SD towards HUVECtert cells were investigated using the LDH and the WST-1 colorimetric assays. The viability of the cells decreased at concentrations greater than $20 \mu \mathrm{M} 4,8$-SD after $6 \mathrm{~h}$ of treatment (Fig. 6). In contrast to 4,8-SD, GluCer were poorly soluble in DMSO and lacked activity in both the ELISA and cell viability studies (data not shown).

\section{Discussion}

We observed that the sphingoid base 4,8-SD of GluCer from A. amurense inhibits the inflammatory responses induced by TNF- $\alpha$ and LPS in HUVECtert cells. The anti-inflammatory effects were observed at significantly lower concentrations of 4,8-SD compared to the cytotoxic effects. The GluCer however lacked effects on the HUVECtert cells in the presented in vitro bioassays. These findings confirm that GluCer may be metabolized to $4,8-\mathrm{SD}$ in the gut lumen by enzymes in order 
to acquire cytotoxic and/or anti-inflammatory properties (5). The daily intake by humans of total SLs from plant sources is estimated to be as little as $50 \mathrm{mg}(9,15)$. It is unknown what portion of 4,8-SD becomes bioavailable in the mucosal cells of the intestines after ingestion of complex SLs. However, it was reported that sphingadienines in general have the advantage over other SLs of being slowly metabolized and of having a long half-life in intestinal epithelial cells (21).

Previously, pro-apoptotic effects of 4,8-SD and various other sphingoid bases were reported which were regulated by activation of caspases thereby explaining the cytotoxic effects (22-25). Although there appears to be a difference between concentrations inhibiting IL-8 and E-selectin production and cytotoxicity, the effects induced by TNF- $\alpha$ and LPS could still be toxic to the cells. Therefore, deeper in vitro and in vivo studies regarding the anti-inflammatory and cytotoxic effects of 4,8-SD are required. Studies on the effect of 4,8-SD on a wider spectrum of pro- (e.g. IL-1 $\beta$, IL-6, IL-12, IFN $\gamma$ ) and anti-inflammatory cytokines and mediators (e.g. IL-4, IL-10, IL-13), will provide insight in the compounds' specificity and sensitivity. Furthermore, the effects of long-term exposure by 4,8-SD on its anti-inflammatory and cytotoxic activities and studies pertaining to the involved molecular mechanisms of this compound are needed.

Taken together, the present findings revealed that $4,8-\mathrm{SD}$ alters the inflammatory responses of endothelial cells in vitro in a favorable way. Since the source of $4,8-\mathrm{SD}$ can be found in the human diet, consecutive studies on the nutritional and therapeutical function of 4,8-SD merit attention.

\section{Acknowledgements}

This study was supported by the Sino-Austrian Research Project (to B.K.) funded by the Austrian Federal Ministry of Science and Research and Federal Ministry of Health, Women and Youth, and NFN-project 'Drugs from Nature Targeting Inflammation - DNTI', Subproject S10713 from the Austrian Science Fund (to V.B.).

\section{References}

1. Bartke $\mathrm{N}$ and Hannun YA: Bioactive sphingolipids: metabolism and function. J Lipid Res (Suppl 50): S91-S96, 2009.

2. Hannun YA and Obeid LM: Principles of bioactive lipid signalling: lessons from sphingolipids. Nat Rev Mol Cell Biol 9: 139-150, 2008 .

3. Tan RX and Chen JH: The cerebrosides. Nat Prod Rep 20: 509-534, 2003

4. El Alwani M, Wu BX, Obeid LM and Hannun YA: Bioactive sphingolipids in the modulation of the inflammatory response. Pharmacol Ther 112: 171-183, 2006.

5. Lahiri S and Futerman AH: The metabolism and function of sphingolipids and glycosphingolipids. Cell Mol Life Sci 64: 2270-2284, 2007.
6. Lynch DV and Dunn TM: An introduction to plant sphingolipids and a review of recent advances in understanding their metabolism and function. New Phytol 161: 677-702, 2004.

7. Coussens LM and Werb Z: Inflammation and cancer. Nature 420: 860-867, 2002.

8. Nilsson A and Duan RD: Absorption and lipoprotein transport of sphingomyelin. J Lipid Res 47: 154-171, 2006.

9. Vesper H, Schmelz EM, Nikolova-Karakashian MN, Dillehay DL, Lynch DV and Merrill AH Jr: Sphingolipids in food and the emerging importance of sphingolipids to nutrition. J Nutr 129: 1239-1250, 1999.

10. Sugawara T, Kinoshita M, Ohnishi M, Nagata J and Saito M: Digestion of maize sphingolipids in rats and uptake of sphingadienine by Caco-2 cells. J Nutr 133: 2777-2782, 2003.

11. Imai H, Ohnishi M, Hotsubo K, Kojima M and Ito S: Sphingoid base composition of cerebrosides from plant leaves. Biosci Biotechnol Biochem 61: 351-353, 1997.

12. Pruett ST, Bushnev A, Hagedorn K, et al: Biodiversity of sphingoid bases ('sphingosines') and related amino alcohols. J Lipid Res 49: 1621-1639, 2008.

13. Nixon GF: Sphingolipids in inflammation: pathological implications and potential therapeutic targets. Br J Pharmacol 158: 982-993, 2009.

14. Jung JH, Lee CO, Kim YC and Kang SS: New bioactive cerebrosides from Arisaema amurense. J Nat Prod 59: 319-322, 1996.

15. Sugawara T and Miyazawa T: Separation and determination of glycolipids from edible plant sources by high-performance liquid chromatography and evaporative light-scattering detection. Lipids 34: 1231-1237, 1999.

16. Hayashi $\mathrm{A}$ and Matsubara $\mathrm{T}$ : Determination of the structure of sphinga-4,8-dienine from oyster glycolipids by gas chromatography and mass spectrometry. Biochim Biophys Acta 248: 306-314, 1971.

17. Schiller HB, Szekeres A, Binder BR, Stockinger H and Leksa V: Mannose 6-phosphate/insulin-like growth factor 2 receptor limits cell invasion by controlling alphaVbeta 3 integrin expression and proteolytic processing of urokinase-type plasminogen activator receptor. Mol Biol Cell 20: 745-756, 2009.

18. Takano H, Murasawa S and Asahara T: Functional and gene expression analysis of hTERT overexpressed endothelial cells. Biologics 2: 547-554, 2008.

19. Pierce JW, Schoenleber R, Jesmok G, et al: Novel inhibitors of cytokine-induced IkappaBalpha phosphorylation and endothelial cell adhesion molecule expression show anti-inflammatory effects in vivo. J Biol Chem 272: 21096-21103, 1997.

20. Bochkov VN, Kadl A, Huber J, Gruber F, Binder BR and Leitinger N: Protective role of phospholipid oxidation products in endotoxin-induced tissue damage. Nature 419: 77-81, 2002.

21. Fyrst H, Oskouian B, Bandhuvula $\mathrm{P}$, et al: Natural sphingadienines inhibit Akt-dependent signaling and prevent intestinal tumorigenesis. Cancer Res 69: 9457-9464, 2009.

22. Ohta H, Sweeney EA, Masamune A, Yatomi Y, Hakomori S and Igarashi Y: Induction of apoptosis by sphingosine in human leukemic HL-60 cells: a possible endogenous modulator of apoptotic DNA fragmentation occurring during phorbol esterinduced differentiation. Cancer Res 55: 691-697, 1995.

23. Park MT, Kang JA, Choi JA, et al: Phytosphingosine induces apoptotic cell death via caspase 8 activation and Bax translocation in human cancer cells. Clin Cancer Res 9: 878-885, 2003.

24. Sugawara T, Zaima N, Yamamoto A, Sakai S, Noguchi R and Hirata T: Isolation of sphingoid bases of sea cucumber cerebrosides and their cytotoxicity against human colon cancer cells. Biosci Biotechnol Biochem 70: 2906-2912, 2006.

25. Aida K, Kinoshita M, Sugawara T, Ono J, Miyazawa T and Ohnishi M: Apoptosis inducement by plant and fungus sphingoid bases in human colon cancer cells. J Oleo Sci 53: 503-510, 2004. 